\title{
PROTOCOLO ANATÔMICO-CIRÚRGICO DAS RELAÇÕES ENTRE OS NERVOS LARÍNGEOS RECORRENTES E AS ARTÉRIAS TIREÓIDEAS INFERIORES: ESTUDO EM 79 TIREOIDECTOMIAS
}

\author{
ANATOMICAL-SURGICAL PROTOCOL OF THE RELATION BETWEEN RECURRENT \\ LARINGEAL NERVES AND INFERIOR THYROIDAL ARTERY IN 79 THYROIDECTOMIES
}

\author{
João Bosco Botelho, TCBC-AM'; Gecildo Soriano dos Anjos² ${ }^{2}$ Givanildo de Pádua Pires ${ }^{3}$; \\ Daniele Memória Ribeiro Ferreira ${ }^{3}$; Tarick de Oliveira Leite ${ }^{4}$; Gilmara Anne da Silva Resende ${ }^{5}$
}

\begin{abstract}
RESUMO: Introdução: Os cuidados com as preservações dos nervos laríngeos recorrentes e das glândulas paratireóides, nas tireoidectomias, continuam desafiando os cirurgiões em razão das graves complicações que podem ocorrer quando são manipulados inadequadamente. $\mathrm{O}$ trabalho teve como objetivo, no curso das tireoidectomias totais e parciais, estabelecer protocolo anatômicocirúrgico das relações anatômicas entre os nervos laríngeos recorrentes com as artérias tireóideas inferiores, identificar-preservar esses nervos e as glândulas partireóides. Método: Os registros fotográficos durante os trans-operatórios foram obtidos de 79 pacientes submetidos às tireoidectomias totais e parciais (lobectomia total direita ou esquerda e istmectomia), respectivamente, com as identificações dos nervos e artérias bi ou unilateralmente, propondo expor as relações anatômicas entre essas estruturas, operados entre janeiro de 2005 e julho de 2006. Resultados: Os registros fotográficos foram adaptados aos desenhos esquemáticos para estabelecer os principais pontos de referências anatômicas dos nervos laríngeos recorrentes em relação às artérias tireóideas inferiores, totalizando 116 nervos laríngeos recorrentes. Conclusão: A íntima relação dos nervos laríngeos recorrentes e as artérias tireóideas inferiores podem se apresentar de diversas formas, incluindo as anomalias congênitas, como a do laríngeo não recorrente, as duplicações e triplicações pré-laríngeas do nervo, sem dúvida, algumas vezes, dificultando a identificação dessas estruturas. São imperativas as claras identificações dessas estruturas e das glândulas paratireóides para preservá-las, no curso das tireoidectomias totais e parciais, a fim de evitar as complicações pós-operatórias (Rev. Col. Bras. Cir. 2007; 34(3): 142-146).
\end{abstract}

Descritores: Nervos laríngeos; Glândula tireóide; Tireoidectomia.

\section{INTRODUÇÃO}

No Brasil, as doenças cirúrgicas da glândula tireóide são muito mais freqüentes nas áreas geográficas bociogênicas, como no Amazonas, e continuam representando importante problema de saúde pública ${ }^{1,2}$. Dessa forma, as indicações cirúrgicas como tratamento preferencial das dismorfias tireóideas, sejam bócios, inflamatórias, tumorais benignas e malignas, fazem parte do cotidiano dos cirurgiões especialistas e gerais.

Em conseqüência das dificuldades ao acesso hospitalar, as pessoas portadoras de doenças da tireóide, em especial as que habitam as áreas distantes dos centros urbanos, no Norte, não raras vezes chegam aos hospitais com bócios de grande volume (BGV), aumentando as dificuldades técnicas da execução das tireoidectomias, e, assim, podendo aumentar o índice de complicações cirúrgicas per e pós-operatórias.
O bócio é considerado de grande volume quando estão presentes três das quatro condições seguintes ${ }^{1,2}$ :

- Cranial: alcança a borda superior da cartilagem tireóidea do laringe;

- Caudal: ocupa a fossa supra-esternal sem penetrar no tórax;

- Lateral direito: desloca anterior e lateralmente a borda médio-ventral do músculo esternocleidomastóideo;

- Lateral esquerdo: desloca anterior e lateralmente a borda médio-ventral do músculo esternocleidomastóideo.

Em consequiência dos muitos vetores oriundos do tripé - condições pré-operatórias e a doença de cada paciente, treinamento das equipes cirúrgicas e o suporte hospitalar que une as práticas cirúrgicas como atividade médica intervencionista, a literatura médica continua expondo os acidentes per e pós-operatórios nas tireoidectomias, capazes de gerar seqüelas funcionais importantes, especialmente nos

1. Professor-Doutor e Livre Docente da Disciplina Otorrinolaringologia-Cirurgia de Cabeça e Pescoço da Universidade do Estado do Amazonas (UEA) e do Centro Universitário Nilton Lins (UNINILTON LINS); Professor Convidado Permanente da Universidade de Paris VII (CHU BichatClaude Bernard) e Professor Titular aposentado da Universidade Federal do Amazonas.

2. Professor-Mestre da Disciplina Otorrinolaringologia-Cirurgia de Cabeça e Pescoço do UNINILTON LINS e orientador das Residências em Otorrinolaringologia do Hospital Santa Júlia (HSJ) e do Hospital Adriano Jorge / UEA.

3. Médico-Residente da Residência em Otorrinolaringologia do HSJ.

4. Acadêmico do $9^{\circ}$ período do Curso de Medicina do UNINILTON LINS e Bolsista da Fundação de Amparo à Pesquisa do Estado do Amazonas.

5. Doutoranda do Curso de Medicina do UNINILTON LINS

Recebido em 16/10/2006

Aceito para publicação em 13/12/2006

Conflito de interesses: nenhum

Fonte de financiamento: nenhuma

Trabalho realizado na Residência Médica em Otorrinolaringologia do Hospital Santa Júlia (Credenciada pelo CNRM-MEC).

$1^{\circ}$ Lugar no Programa de Iniciação Científica 2005-2006, do Centro Universitário Nilton Lins - Fundação de Amparo à Pesquisa do Estado do Amazonas. 
$\mathrm{BGV}$, relacionadas com o manuseio cirúrgico dos nervos laríngeos recorrentes (NLRs), artérias tireóideas inferiores (ÁTIS), e as glândulas paratireóides (GPs) ${ }^{3-6}$.

Este trabalho está inserido na perspectiva de contribuir para organizar um protocolo anatômico-cirúrgico a fim de evitar essas complicações, voltado às características clínicocirúrgicas dos bócios diagnosticados no nosso país, notadamente no Norte, sem dúvida com tamanhos maiores se comparados às doenças da tireóide de outros centros urbanos no Brasil e no exterior ${ }^{1,2,6,7}$.

\section{MÉTODO}

A coleta de dados foi realizada entre janeiro de 2005 e junho de 2006, no Serviço de Otorrinolaringologia e Cirurgia de Cabeça e Pescoço - Residência Médica em Otorrinolaringologia, do Hospital Santa Júlia, Manaus, em 79

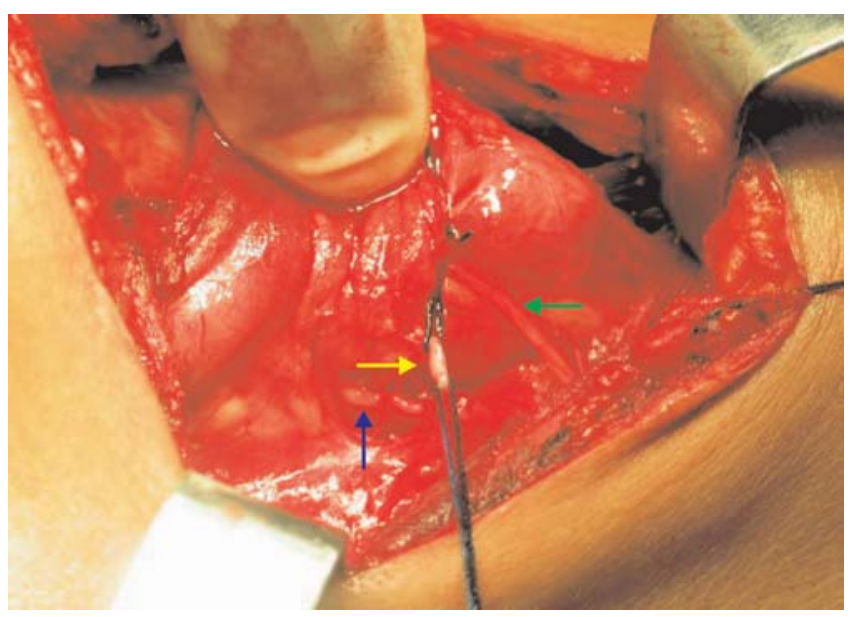

Figura 1 - Nervo laríngeo recorrente com um único pré-laríngeo: 109 pacientes $=94 \%$.
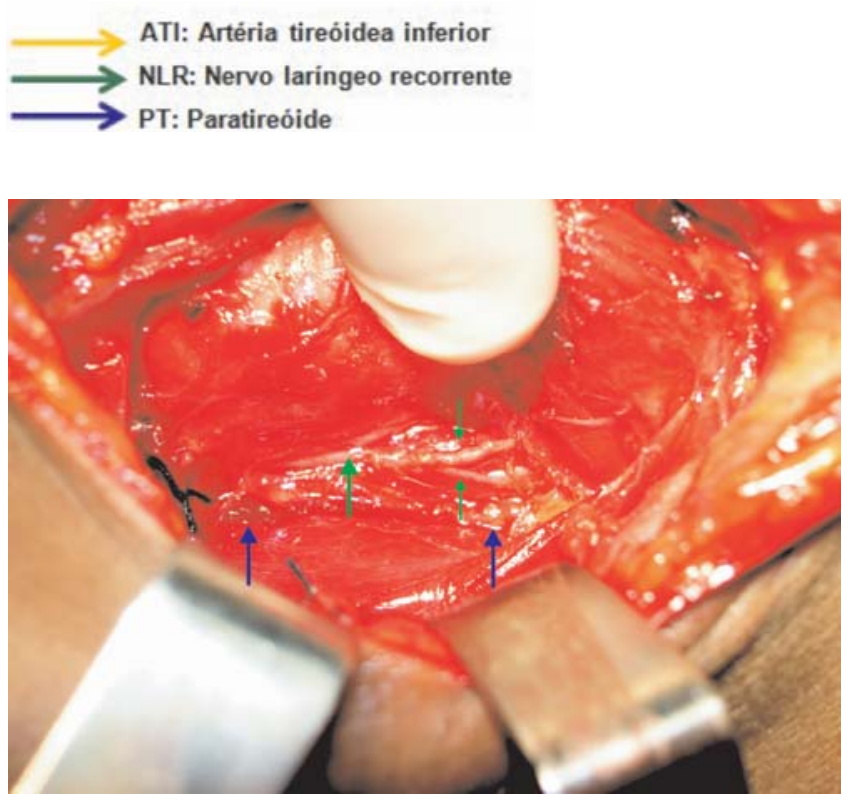

Figura 2 - Nervo laríngeo recorrente bifurcado pré-laríngeo: 3 pacientes $=2,6 \%$

$\longrightarrow$ NLR: Nervo laríngeo recorrente pré-laríngeo duplicado PT: Paratireóide pacientes submetidos as tireoidectomias totais e parciais, estas últimas sempre representadas pelas lobectomias totais direita ou esquerda e istmectomia.

Os registros fotográficos mostraram diferentes etapas cirúrgicas das tireoidectomias parciais e totais, em especial, as relações anatômicas entre os NLRs e as ATIs, além de identificar e preservar os NLRs e as GPs.

Critérios de inclusão: pacientes portadores de bócios, processos inflamatórios, tumores benignos e malignos com indicação cirúrgica de tireoidectomia total ou parcial.

Critérios de exclusão: pacientes que foram submetidos à tireoidectomia total ou parcial sem registro fotográfico e os que as imagens não ficaram claras para a identificação das estruturas anatômicas estudadas.

\section{RESULTADOS}

O total de 79 pacientes foi submetido à tireoidectomia total ou parcial (lobectomia total direita ou esquerda e istmectomia). Desse universo, 28 tireoidectomias parciais foram realizadas, sendo 17 à esquerda e 11 à direita, e as tireoidectomias totais em 51 pacientes.

O trabalho obteve imagens de 116 NLRs com as seguintes características:

- NLR com um único ramo pré-laríngeo (Figura 1): 109 $=94 \%$;

- NLR bifurcado pré-laríngeo (Figura 2), 2 à esquerda e 1 à direita, $3=2,6 \%$;

- NLR trifurcado pré-laríngeo (Figura 3), 1 com variação anatômica bilateral e 1 à esquerda: $3=2,6 \%$;

- NL não recorrente (Figura 4): $1=0,8 \%$.

Em relação às relações anatômico-cirúrgicas entre os NLRs e a ATIs foram registradas (Figura 5):

1 NLR passando a frente da ATI à direita: $4(3,45 \%)$;

2 NLR passando a frente da ATI à esquerda: $4(3,45 \%)$;

3 NLR passando atrás da ATI à direita: $45(38,80 \%)$;

4 NLR passando atrás da ATI à esquerda: 51 (43,97\%);

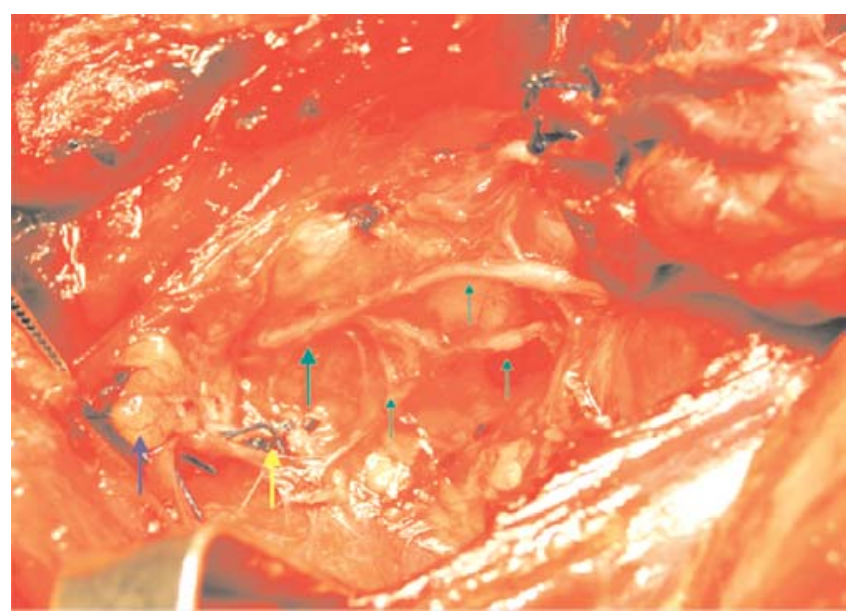

Figura 3 - Nervo laríngeo recorrente trifurcado pré-laríngeo: 3 pacientes $=2,6 \%$.

ATI: Artéria tireóidea inferior ligada entre fios

$\longrightarrow$ NLR: Nervo laringeo recorrente triplicado pré-laringeo PT: Paratireóide 


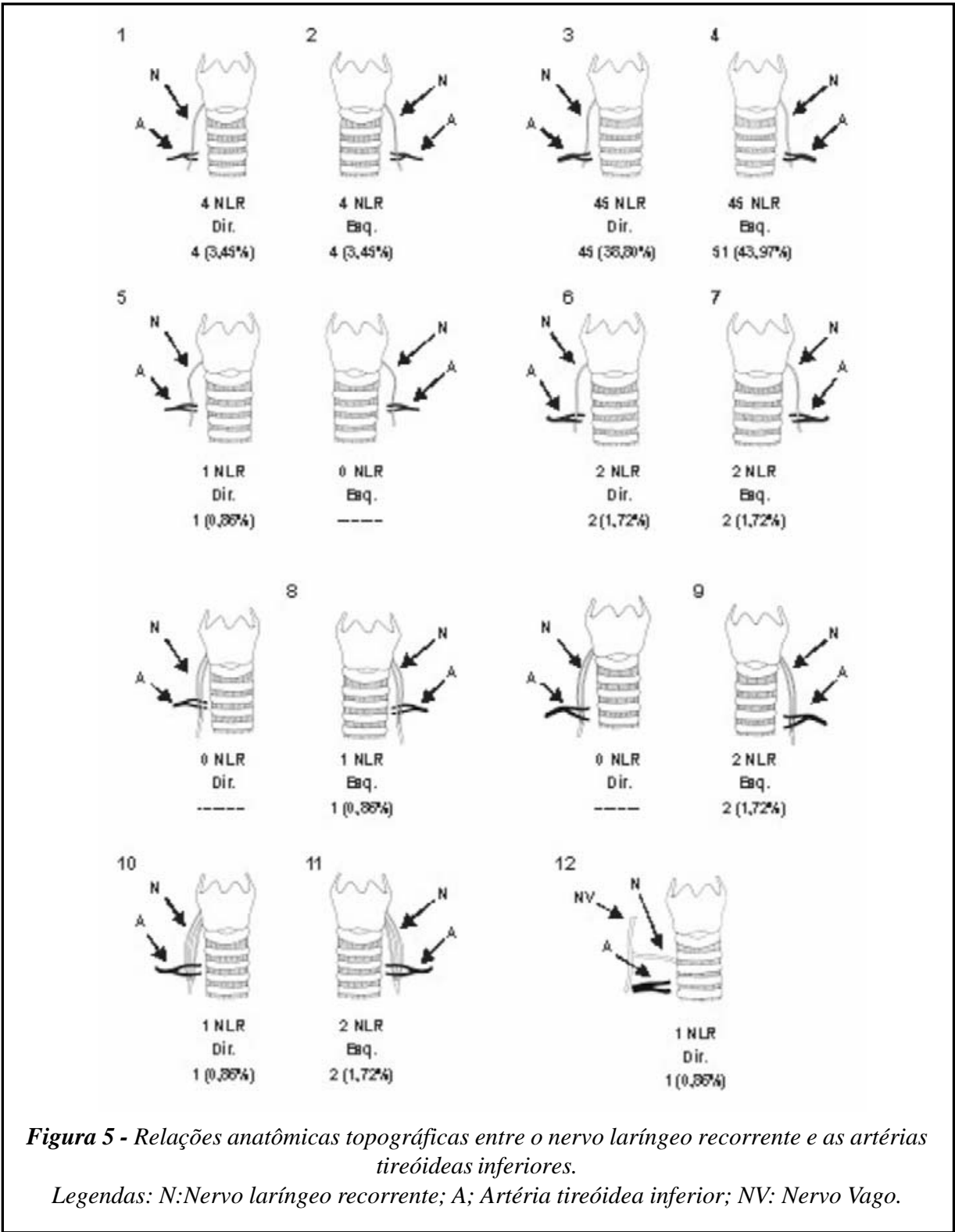

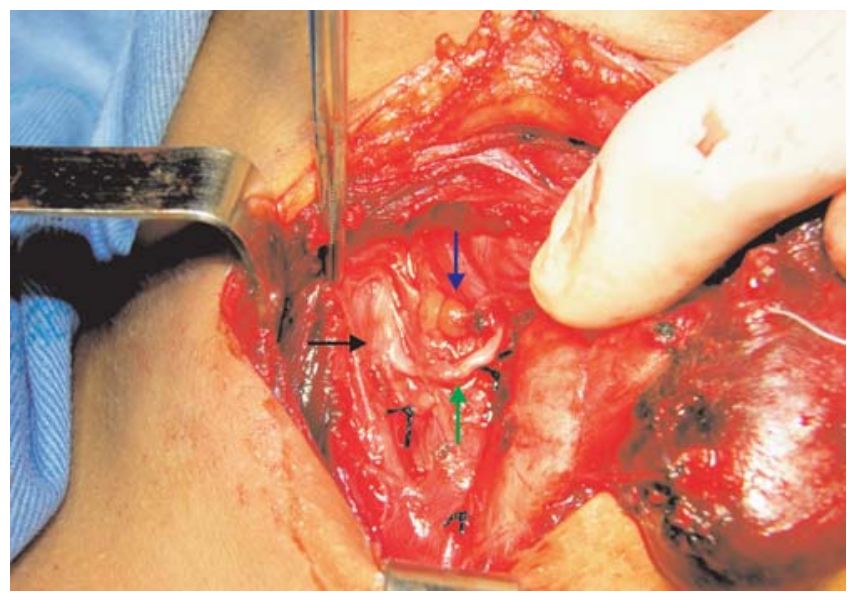

Figura 4 - Nervo laríngeo não recorrente: 1 paciente $=0,8 \%$.

$\longrightarrow N L R:$ Nervo laringeo não recorrente

PT: Paratireóide

NV: Nervo vago
5 NLR passando a frente do ramo inferior da ATI à direita: $1(0,86 \%)$;

6 NLR passando a frente do ramo superior da ATI à direita: $2(1,72 \%)$;

7 NLR passando a frente do ramo superior da ATI à esquerda: $2(1,72 \%)$;

8 NLR bifurcado passando a frente da ATI à esquerda: $1(0,86 \%)$;

9 NLR bifurcado passando atrás da ATI à esquerda: 2 $(1,72 \%)$;

10 NLR trifurcado passando atrás da ATI à direita: 1 $(0,86 \%)$;

11 NLR trifurcado passando atrás da ATI à esquerda: $2(1,72 \%)$.

12 NL não recorrente, saindo diretamente do nervo vago à direita, por meio de concavidade cranial, ao nível do terceiro anel traqueal, sem relação próxima com a AT: 1 $(0,86 \%)$. 


\section{DISCUSSÃO}

Vários procedimentos cirúrgicos já foram descritos como sendo a determinante de lesão dos NLRs: tireoidectomias parciais e totais, paratireoidectomias, esofagectomias, pneumectomias, traqueoplastias, entre outras. Sem dúvida, as tireoidectomias constituem a mais freqüente causa de injúria aos NLRs ${ }^{8,9,10}$.

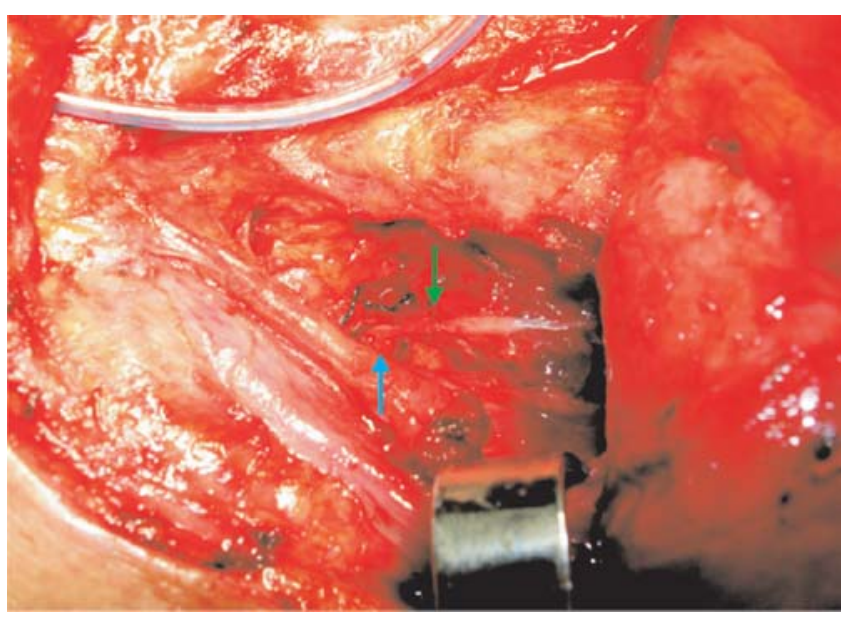

Figura 6 - Neurorrafia término-terminal da lesão do nervo laríngeo recorrente em bócio com componente mediastínico.

Neurorrafia término-terminal do nervo laringeo recorrente

Glândula paratireóide

Reed $^{11}$ no mais importante trabalho sobre as variações anatômicas dos NLRs, citado até hoje, descreveu 28 diferentes tipos de relação entre os NLRs e a ATIs, classificando-as em três tipos básicos: Nervo laríngeo anterior à artéria tireóidea inferior; Nervo laríngeo posterior à artéria tireóidea inferior; Nervo laríngeo entre os ramos da artéria tireóidea inferior.

Diferente dos estudos das variações anatômicas dos NLRs, realizados nos grandes centros urbanos, onde o tempo transcorrido entre a indicação cirúrgica e a execução da tireoidectomia é cada vez menor, no Amazonas, os pacientes oriundos dos confins da floresta portadores de BGV fazem parte do cotidiano, com algumas peças cirúrgicas pesando entre setenta e trezentos gramas ${ }^{1,2,6,7,8}$. Nesses pacientes com componente mediastínico do bócio, nos quais a exérese pode impor ou não a esternotomia segmentar, e mais raramente, a completa osteotomia do esterno, mesmo com os rigores da técnica cirúrgica, a identificação anatômica do NLR fica dificultada pelo espaço exíguo no qual o cirurgião trabalha. Nas exéreses da porção torácica do bócio por via transcervical, procedimento que evita a esternotomia, a manobra do cirurgião com os dedos no mediastino anterior em torno do bócio, sem visão direta, para movê-lo em direção cranial, pode ocorrer a lesão do NLR ${ }^{1,2,7,8,10,12-15}$. Por outro lado, nesses casos, se a hemostasia está acoplada aos rigores da diérese apropriada, com as artérias tireóideas identificadas e ligadas e sem sangramento fora de controle, quase sempre é possível a identificação dos cotos do NLR lesado e, dessa forma, imediatamente, proceder a neurorrafia término-terminal ${ }^{9}$ (Figura 6). Em outros, nos tumores malignos da tireóide com extensão extra-capsular e algumas tireoidites, mesmo com as ligaduras vasculares realizadas, a aderência aos músculos infra-hióideos, em especial ao esterno-tireóideo, para manter a radicalidade oncológica, nem sempre é possível preservar o NLR ${ }^{16}$ (Figura 7).

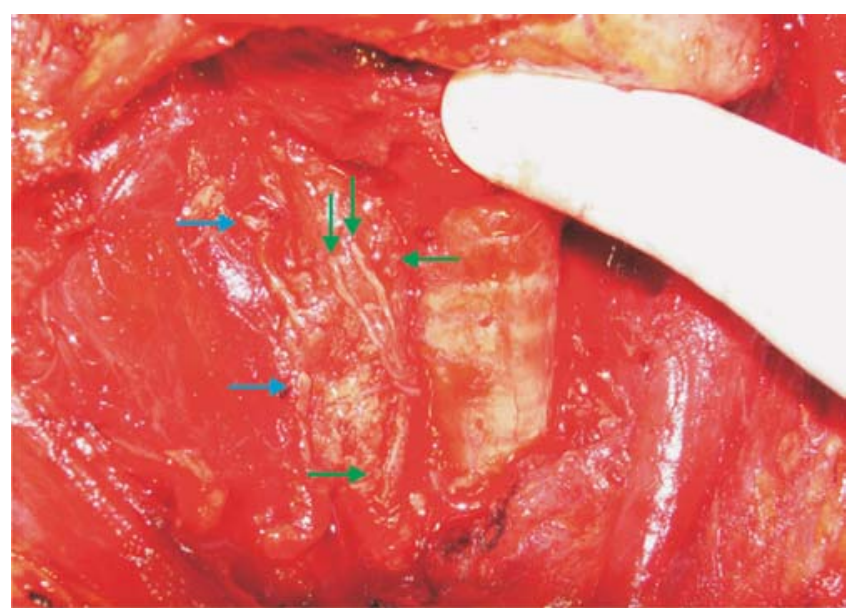

Figura 7 - A secção cirúrgica deliberada, na tireoidectomia total, de dois dos três ramos do nervo laríngeo recorrente direito trifurcado pré-laríngeo, para manter a radicalidade oncológica, mantendo a integridade da porção mais espessa do nervo, em paciente do sexo feminino, 56 anos, com diagnóstico pré-operatório pela PAAF de adenocarcinoma papilifero.

$\longrightarrow$ NLR: Nervo laringeo recorrente com dois dos três ramos seccionados PT: Paratireóides

Sem poder isolar a importância da identificação e preservação das GPs desse conjunto anatômico-cirúrgico, mesmo com as variações de forma, tamanho e localização, as claras identificações dos NLRs e das ATIs facilitam enormemente a preservação das GPs $^{3-5,10,16-18}$.

Salvo as anomalias congênitas, como os três NLRs duplicados, os três triplicados no trajeto pré-laríngeo e um laríngeo não recorrente, isto é, o nervo faz curva de concavidade cranial ao nível do segundo anel traqueal e penetra diretamente na laringe, os resultados deste trabalho mostram concordância com a literatura consultada, enfatizando que dos 116 NLRs identificados, 97 (84\%) deles passavam atrás das ATIs, sendo 51 à esquerda e 46 à direita. 


\begin{abstract}
Background: Care in preserving the recurrent laryngeal nerves and the parathyroids glands, during thyroidectomies, continue to challenge surgeons because of the serious complications, which can occur when they are inadequately handled. The work aimed at establishing a surgical-anatomical protocol of the relations between the recurrent laryngeal nerves with the lower thyroidal arteries and identify and preserve the nerves and the parathyroids glands, in the course of total and partial thyroidectomies. Methods: The photographic records during the surgery have been obtained from 79 patients who were submitted to total and partial thyroidectomies (total right or left lobectomy and istmectomy) respectively, with identification of the nerves and arteries, both unilateral and bilateral, proposing to expose the anatomical relations between these structures, operated on between January 2005 and July 2006. Results: The photographic records were adapted to the schematic drawings so as to establish the major anatomical points of reference of the recurrent laryngeal nerves vis-à-vis the inferior thyroidal arteries were completed in a total of 116 recurrent laryngeal nerves. Conclusion: The intrinsic relation of the nerves with the arteries can occur in different forms, including the congenital abnormalities, as that of the non-recurrent laryngeal, the prelaryngeal duplications and triplications of the nerve, undoubtedly hindering the identification of such structures. Even under the protection of the anatomic and surgical difficulties, a clear identification of the nerve, artery and the parathyroid glands is imperative to preserve them in the course of total and partial thyroidectomies in order to avoid post-surgical complications.
\end{abstract}

Key words: Laryngeal Nerves; Thyroid gland; Thyroidectomy.

\section{REFERÊNCIAS}

1. Botelho JB. Patologia da glândula tireóide. In: Botelho JB. Otorrinolaringologia e cirurgia de cabeça e pescoço para estudantes. Manaus: Editora da Universidade Federal do Amazonas; 2000. p. 325-98

2. Botelho JB. Pathologie de la glande thyroïde. In: Botelho JB, Gehanno P. Otorhinolaryngologie et chirurgie cervico-faciale à 1'usage dês étudiants. Paris: Edk; 2002. p. 61-90.

3. Bliss RD, Gauger PG, Delbridge LW. Surgeon's approach to the thyroid gland: surgical anatomy and the importance of technique. World J Surg. 2000; 24(8):891-7.

4. Gouch IR, Wilkinson D. Total thyroidectomy for management of thyroid disease. World J Surg. 2000; 24(8):962-5.

5. Reeve T, Thompson NW. Complications of thyroid surgery: how to avoid them, how to mange them, and observations on their possible effect on the whole patient. World J Surg. 2000; 24(8):971-5.

6. Botelho JB, Cattebeki L, Cattebeki T, Anjos GS, Lima RM, Barbosa MF, Aguiar GC. Histopathologie de 573 thyroïdectomies réalisées entre 1976 et 1998 Manaus - Brésil. Revue Officielle de La Societé Française D'ORL et de Chirurgie de La Face et Cou. Paris. 2001;66(2):47-49.

7. Ceballos RG. Comportamiento de los bocios de gran volumen en la ciudad de Manaus [dissertação]. Manaus (AM): Universidade Federal do Amazonas; 2000.

8. Botelho JB. Complicações das tireoidectomias - lesão do nervo laríngeo recorrente. Anais do $25^{\circ}$ Congresso Brasileiro de Cirurgia; 2003 Jul 6-10; Porto Alegre; Brasil; 2003.

9. Monfared A, Gorti G, Kim D. Microsurgical anatomy of the laryngeal nerves as related to thyroid surgery. Laryngoscope. 2002; 112(2):386-92.

10. Hartl DM, Brasnu D. Les paralysies récurrentielles: connaissances actuelles et traitements. Ann Oto-laryngol Chir Cervico-fac. 2000; 117(2):60-84.

11. Reed AF. The relations of the inferior laryngeal nerve to the inferior thyroid artery. Anat Rec. 1943; 185(1):17-23.
12. Monchik JM, Materazzi G. The necessity for a thoracic approach in thyroid surgery. Arch Surg. 2000; 135(4):467-71; discussion 471-2.

13. Shai SE, Chen CY, Hsu CP, Hsia JY, Yang SS, Chuang CY, Wang PY. Surgical management of substernal goiter. J Formos Med Assoc. 2000; 99(11):827-32.

14. Mussi A, Ambrogi MC, Iacconi P, Spinelli C, Miccoli P, Angeletti CA. Mediastinal goitres: when the transthoracic approach? Acta Chir Belg. 2000; 100(6):259-63.

15. Arici C, Dertsiz L, Altunbas H, Demircan A, Emek K. Operative management of substernal goiter: analysis of 52 patients. Int Surg. 2001; 86(4):220-4.

16. Manolidis S, Takashima M, Kirby M, Scarlett M. Thyroid surgery: a comparison of outcomes between experts and surgeons in training. Otolaryngology Head Neck Surg. 2001; 125(1):30-33.

17. Botelho JB, Cançado AR, Sousa EA. Características macroscópicas, localização e suprimento vascular das glândulas paratireóides cervicais. Prêmio Nacional de Anatomia Humana 2002 Prof. Garcia do Prado. Rio Grande do Sul: Pontífice Universidade Católica; 2002. 230 p.

18. Botelho JB, Cançado AR, Sousa EA. A Importância anatomocirúrgica das características macroscópicas, localização e suprimento vascular das glândulas paratireóides cervicais. Rev Col Bras Cir. 2004; 31(2):132-8.

Como citar este artigo:

Botelho JB, Anjos GS, Pires GP, Ferreira DM, Leite TO, Resende GA. Protocolo anatômico-cirúrgico das relações entre os nervos laríngeos recorrentes e as artérias tireóideas inferiores: estudo em 79 tireoidectomias. Rev Col Bras Cir [periódico na Internet]. 2007;34(3). Disponível em URL: http://www.scielo.br/rcbc

Endereço para correspondência:

João Bosco Botelho

Av. Eduardo Ribeiro, 520 - sala 705

69010-010 - Manaus - AM

joão_botelho@vivax.com.br

joaoboscobotelho@gmail.com 